\title{
CONOCIMIENTO ESPECIALIZADO PARA ENSEÑAR LA OPERACIÓN DE RESTA EN EDUCACIÓN INFANTIL
}

\section{KINDERGARTEN TEACHERS' SPECIALISED KNOWLEDGE ON SUBTRACTION}

\section{CONHECIMENTO ESPECIALIZADO DO PROFESSOR DA EDUCAÇÃO INFANTIL NO ÂMBITO DO TEMA DA SUBTRAÇÃO}

\author{
M. Cinta Muñoz-Catalán \\ Profesora Doctora en la Facultad de Ciencias de la Educación de la \\ Universidad de Sevilla. Espanha. \\ mcmunozcatalan@us.es \\ María del Mar Liñán García \\ Profesora Doctora en la Facultad de Ciencias de la Educación de la \\ Universidad de Sevilla y del Centro de Estudios Universitarios \\ Cardenal Spínola CEU. Espanha \\ mlinan@us.es \\ Miguel Ribeiro \\ Profesor Doctor en la Facultad de Educación de la \\ Universidad Estadual de Campinas (UNICAMP). São Paulo-SP, Brasil. \\ cmribas78@gmail.com
}

\begin{abstract}
Resumen: En este trabajo reflexionamos sobre el conocimiento profesional del profesor de Educación Infantil en relación con la resta. La atención al conocimiento deseable de este profesional es relativamente reciente, existiendo escasos estudios que aborden este tema. Partimos de la consideración de que este conocimiento es especializado; diferente al del profesor de Primaria o Secundaria; y su caracterización debe realizarse desde un enfoque centrado en la propia matemática. Esto nos lleva a considerar el modelo del Conocimiento Especializado del Profesor de Matemáticas (Mathematics Teachers' Specialised Knowledge, MTSK) como herramienta teórica y analítica idónea para ofrecer una propuesta de elementos de conocimiento especializado que, a la luz de nuestra experiencia docente e investigadora, consideramos que deseables para este profesional en relación con la resta, incluyendo aspectos de conocimiento del contenido (Mathematical Knowledge, MK) y de conocimiento didáctico del contenido (Pedagogical Content Knowledge, PCK). La reflexión sobre esta propuesta nos lleva a destacar tres elementos principales que parecen caracterizadores de la naturaleza de este conocimiento: su densidad y cohesión, la profundidad de los conocimientos matemáticos implicados y su repercusión para construir un adecuado PCK; y la relevancia del conocimiento de las fases que los niños siguen en su proceso de comprensión del número. Se aportan sugerencias para la formación inicial y continua de estos profesionales.
\end{abstract}

Palabras clave: Conocimiento especializado del profesor de matemáticas. MTSK. Profesor de Educación Infantil. Resta. Conocimiento matemático del contenido y conocimiento didáctico del contenido.

Abstract: In this paper we reflect upon the early childhood teachers' professional knowledge in relation to subtraction. Paying attention to this professional desirable knowledge is relatively recent, and thus there are few studies that address it. We assume as a starting point the consideration that this knowledge is specialized; differs from that of primary or secondary teachers'; and its characterization must be carried out from an approach centered on mathematics itself. This leads us to consider the model of Mathematics Teachers' Specialised Knowledge (MTSK) as a suitable theoretical and analytical tool to offer a proposal of specialized knowledge elements that, in light of our teaching and research experience, we considered desirable for the work of teaching of these professionals in relation to subtraction. In this proposal we include aspects of Mathematical Knowledge (MK) and of Pedagogical Content knowledge (PCK). In the proposal we highlight three main elements that seem to characterize the nature of this knowledge: its density and cohesion; the depth of the mathematical knowledge involved and its impact to build an adequate PCK; and the relevance of knowledge of the phases that children follow in their process of understanding the number. Some recommendations for early years teachers' education are provided.

Keywords: Mathematics Teachers' Specialised Knowledge. MTSK. Early childhood teacher. Subtraction. Mathematical knowledge. Pedagogical content knowledge.

Resumo: Neste trabalho, refletimos sobre o conhecimento profissional do professor de Educação Infantil em relação à subtração. Considerar como foco de atenção o conhecimento desejável destes professores é algo relativamente recente, existindo poucos estudos que abordam esta questão. Partimos da consideração de que esse conhecimento é 
especializado; diferente do conhecimento do professor das etapas escolares seguintes; e sua caracterização deve ser realizada a partir de uma abordagem centrada na própria matemática. Nesse sentido consideramos a conceitualização do Mathematics Teachers' Specialized Knowledge (MTSK) como uma ferramenta teórica e analítica para conceitualizar uma proposta de elementos constituintes do conhecimento especializado ideal do professor no âmbito que, à luz de nossa experiência de ensino e pesquisa, consideramos desejável para este profissional em relação à subtração. Nesta proposta incluímos aspectos do conhecimento do conteúdo e do conhecimento pedagógico do conteúdo (PCK). Nesta proposta destacamos três elementos principais que parecem caracterizar a natureza desse conhecimento: sua densidade e coesão; a profundidade do conhecimento matemático envolvido e seu impacto para construir um PCK adequado; e a relevância do conhecimento do professor das fases que as crianças seguem no seu processo de compreensão do número em etapas educativas posteriores. São fornecidas sugestões para a formação inicial e contínua de professores.

Palavras chave: Conhecimento Especializado em Professores de Matemática. MTSK. Professor de Educação Infantil. Subtração. Conhecimento matemático do conteúdo e conhecimento pedagógico do conteúdo.

\section{INTRODUCCIÓN: antecedentes y motivación del estudio}

La investigación actual está poniendo de relieve lo que ya percibíamos como formadores de profesores: la importancia de que los profesores tengan un profundo conocimiento de las matemáticas que enseñan para favorecer un buen aprendizaje matemático de sus estudiantes (e.g., CONTRERAS et al., 2012; HILL; ROWAN; BALL, 2005; MA, 1999). En los últimos treinta años uno de los focos de atención en Educación Matemática ha sido la discusión sobre el conocimiento que puede ser útil para el profesor que enseña matemáticas, que se ha reflejado en diversos modelos de análisis de conocimiento, cada uno de los cuales subraya aspectos particulares de la naturaleza de dicho conocimiento (CARRILLO et al., 2017). La mayoría de estos modelos parten del trabajo de Shulman (1986; 1987), quien planteó la necesidad de considerar la especificidad del contenido que se está enseñando, instando a enfocar el conocimiento necesario para enseñar a través de la lente de la propia disciplina. Introdujo la distinción entre conocimiento de la materia (MK) y conocimiento didáctico del contenido (PCK), entendiendo esta última categoría como la combinación entre contenido y pedagogía y considerándola como la que podía distinguir la comprensión del especialista en contenido de la del pedagogo.

El interés por el conocimiento profesional que debe tener un profesor de Educación Infantil, respecto de la enseñanza de las matemáticas, es relativamente reciente. Los distintos modelos de análisis de este conocimiento se han desarrollado teniendo como referente a profesores de Educación Primaria y Educación Secundaria y poco se sabe todavía sobre la naturaleza particular del conocimiento del profesor de Infantil. Los escasos estudios que hay al respecto se han centrado principalmente en aspectos relacionados con el PCK matemático (e.g., LEE, 2010; MCCRAY; CHEN, 2012), tratando de evidenciar su influencia directa en la calidad instruccional y en el aprendizaje de los alumnos. Comienzan a emerger estudios que abogan por atender también al conocimiento de la propia matemática como elemento necesario para que los profesores construyan un adecuado PCK matemático, demostrando, incluso, su papel en la predicción de la sensibilidad de los profesores hacia la matemática presente en los juegos de los niños de esta etapa (Opperman, Anders y Hachfeld, 2016).

Esta escasa atención al profesor de Educación Infantil contrasta con el compromiso adoptado por diversas asociaciones americanas, tanto específicas de matemáticas como de Educación Infantil (NAEYC; NCTM, 2002, NATIONAL COUNCIL OF TEACHERS OF MATHEMATICS, 2000), por proporcionar unos estándares específicos apoyados en la investigación, que contribuyan a otorgar solidez a las matemáticas de esta etapa educativa, basándose en su rol potencial en aprendizajes futuros. Parks y Wager (2015) se lamentan de que el discurso profesional presente en los artículos de cuatro de las revistas más relevantes para el área (Early Childhood Readearch Quarterly, The Journal of Early Childhood Teacher Education, the Journal of Research in Mathematics Education y The Journal of Mathematics Teacher Education), durante los últimos 20 años no recojan el conocimiento, las habilidades y las disposiciones particulares que requiere un profesor de esta etapa. Así, al no disponer de una comprensión compartida de qué y cómo formar a los maestros de Educación Infantil, se corre el riesgo de que estos implementen una instrucción simplista que enfatice prácticas mecánicas y memorización de conceptos particulares (los que asumen que los 
alumnos deberían aprender en primaria) o que desarrollen una instrucción que enfatice los juegos sin intencionalidad matemática.

En este artículo pretendemos aportar elementos que contribuyan a caracterizar la naturaleza de este conocimiento del profesor de Educación Infantil para desarrollar conocimientos y habilidades matemáticas en sus alumnos. Partimos, por un lado, de la base de que este conocimiento ha de ser especializado, pues supone un modo de conocer la matemática como objeto de enseñanza y aprendizaje que es específico del profesor. Además, para el caso del profesor de infantil, tanto por el tipo de contenido matemático como por las características especiales de los alumnos de esta etapa, coincidimos con Jakobsen, Thames y Ribeiro (2013) en que su conocimiento es diferente al del profesor que enseña en Primaria o Secundaria y que dicho conocimiento para enseñar Números y Operaciones es distinto, por ejemplo, del implicado en la enseñanza de Geometría o Magnitudes. En tercer lugar, consideramos que la caracterización de este conocimiento del profesor debe realizarse desde un enfoque centrado en la propia matemática. La naturaleza abstracta de los contenidos matemáticos posee repercusiones específicas en su aprendizaje y enseñanza en esta etapa lo que nos lleva a centrarnos solo en aquellos elementos en los que el contenido matemático está implicado, sin por ello no considerar aspectos más pedagógicos generales. Por todo ello, asumimos la conceptualización del conocimiento del profesor contenido en el modelo analítico Conocimiento Especializado del Profesor de Matemáticas (por sus siglas en inglés Mathematics Teachers' Specialised Knowledge - MTSK ${ }^{1}$ ) (CARRILLO et al., 2013; CARRILLO et al., 2017; MUÑOZCATALÁN et al., 2015). Al haberse construido sobre la base de estudios con profesores de niveles educativos posteriores, nos interesa ver su utilidad para definir el conocimiento del profesor de Educación Infantil y su naturaleza.

En la naturaleza de este conocimiento hay que identificar los retos particulares que debe asumir el profesor de esta etapa. Por un lado, el profesor ha de ser consciente de que a pesar de que el repertorio matemático en Educación Infantil sea limitado, se pueden generar obstáculos didácticos que son muy persistentes y repercuten en la adquisición de conocimientos en etapas posteriores. $A$ la necesidad de preverlos y prevenirlos se une también la distancia que en esta etapa existe entre el conocimiento matemático consciente, formal y riguroso que debe tener el profesor y el envoltorio lúdico de las tareas de enseñanza según prescripciones curriculares, sin que por ello se diluyan las raíces matemáticas del contenido que se está trabajando. Estas tareas se convierten en experiencias matemáticas, no solo porque desarrollen exclusivamente contenidos matemáticos, sino porque el profesor, con su gestión, ha permitido que los alumnos desarrollen conocimiento prematemático y matemático a lo largo de la misma, a la misma vez que ha conseguido promover simultáneamente un desarrollo intelectual, social, emocional y físico. Asimismo, puesto que se trata de una etapa educativa generadora de lenguaje, el profesor de Infantil debe prestar especial atención a aspectos relacionados con la comunicación matemática del aula, velando por respetar la validez y corrección de los contenidos matemáticos mediante un lenguaje matemático preciso, adecuado y generador de significados.

Thames y Ball (2010) defienden que los profesores requieren un buen conocimiento matemático, pero las definiciones actuales sobre cómo ha de ser este conocimiento resultan aún imprecisas. Se hace imprescindible, por tanto, discutir cuáles son sus características definitorias $y$, por otro lado, cuáles son las relaciones entre el conocimiento necesario y las oportunidades de aprendizaje (HIEBERT; GROUWS, 2007) que ese conocimiento permitirá promover. Comenzamos a acometer esta tarea de definición del conocimiento especializado del profesor de Educación Infantil centrándonos en la resta porque es uno de los temas con el que los alumnos presentan serias dificultades en Primaria (KAMII; LEWIS; KIRKLAND, 2001). Los profesores han de cuestionar este contenido para su aprendizaje, han de reconocer la complejidad inherente a este concepto y ampliar su noción de resta, trascendiendo su mero significado algorítmico, e identificando los contenidos y procedimientos matemáticos y pre-matemáticos que están en la base de la resta, así como los contenidos matemáticos de etapas posteriores a los que servirá de sustento. Esta información no está presente en la mayoría de los currículos oficiales (cuando los hay), pues en ellos, la información correspondiente a los contenidos matemáticos a impartir, a su secuenciación por ciclos y a las orientaciones metodológicas específicas aparecen diluidos. La formación inicial universitaria de estos profesionales en España, a semejanza de otros países (como Brasil) es muy escasa, reduciéndose 1 Hemos optado por mantener el nombre en inglés además del castellano, ya que esta es una conceptualización del conocimiento del profesor reconocida internacionalmente. (véase Figura 1). 
a 6 créditos obligatorios, de los 240 créditos totales, no siempre impartidos por especialistas en el área. Por otro lado, según constatan Parks y Wager (2015) en relación con el bloque de números y operaciones, en el discurso de los artículos consultados específicos del área y de la etapa educativa, se percibe un gran énfasis en la componente procedimental de las operaciones, mientras que está ausente cuál es el contenido matemático apropiado para esta etapa, cómo se deben enseñar y las destrezas numéricas tempranas previas a las operaciones.

Así, a la luz de este panorama, la responsabilidad de qué y cómo enseñar matemáticas recae en un profesional con escasos criterios para afrontarla y que habitualmente presenta importantes carencias en contenidos matemáticos (e.g., MARTINS; RIBEIRO, 2013), muchas de las cuales son el origen de las dificultades que presentan los alumnos. Aunque comienzan a aparecer investigaciones sobre el contenido del conocimiento del profesor para la enseñanza en Infantil, en nuestra revisión no hemos encontrado trabajos que se centren en la comprensión del sentido de la operación resta. Por tanto, como investigadores y formadores de estos profesionales, comenzamos en Ribeiro, Muñoz-Catalán y Liñán (2015) a afrontar el compromiso de elaborar una propuesta de elementos de conocimiento especializado que estimulara la discusión y la investigación en el área. En esta ocasión hemos profundizado y extendido nuestra comprensión de ese conocimiento incorporando elementos no solo del Conocimiento Matemático (MK, nuestro enfoque anterior) sino también del Conocimiento Didáctico del Contenido (PCK). En el siguiente apartado presentamos el modelo analítico del MTSK (CARRILLO et al., 2017; CARRILLO et al., 2013; MUÑOZ-CATALÁN et al., 2015) con el que hemos articulado la reflexión acerca de los elementos de conocimientos deseables para enseñar la resta, tomando en consideración también la comprensión amplia y completa de futuro que los alumnos deben poseer sobre los algoritmos, que les lleve a comprender el significado de los pasos ocultos, de las propiedades matemáticas que los sustentan y de las situaciones a las que la resta las resuelve.

\section{LENTES TEÓRICAS}

El conocimiento de las matemáticas escolares de un profesor competente ha de ser lo suficientemente amplio y profundo para que le permita conceptualizar el contenido que imparte, representarlo de diversas formas y conocer y seleccionar sus conexiones con otros contenidos del mismo nivel, así como con aquellos que suponen una complejización o simplificación del mismo (e.g., SCHOENFELD; KILPATRICK, 2008). Ese conocimiento les permitirá organizar correctamente los contenidos, estructurándolo a través de las grandes ideas matemáticas (CLEMENTS, 2004) y posibilitando un aprendizaje significativo en los alumnos que les permita seguir construyéndolo en etapas posteriores sobre una base sólida.

Es notoria, en consecuencia, la influencia que tiene el conocimiento matemático de un profesor de Infantil, tanto por lo que en el aprendizaje de sus alumnos puede proyectar para el éxito o no en etapas posteriores, como por los posibles obstáculos que se pueden generar en este periodo y que pueden marcar los siguientes. Esto nos plantea la necesidad de preguntarnos cuál ha de ser ese conocimiento matemático, aquello que le va a permitir decidir sobre los mejores abordajes para potenciar un conocimiento significativo generador de conexiones entre conocimientos para aprendizajes futuros. Así, partiendo de que la influencia del conocimiento del profesor de matemáticas en la transición del estudiante de Primaria a Secundaria es fundamental (FERNÁNDEZ; FIGUEIRAS, 2010), consideramos esta idea extensible al conocimiento del profesor de Infantil para, en este caso, favorecer una transición suave y fructífera del alumno a la siguiente etapa, dejando, por lo tanto, la puerta abierta para los aprendizajes futuros.

Consideramos en este trabajo el modelo del Conocimiento Especializado del Profesor de Matemáticas (Mathematics Teachers' Specialised Knowledge, MTSK) (CARRILLO et al., 2017; CARRILLO et al., 2013; MUÑOZ-CATALÁN et al., 2015). Este modelo considera la especialización como eje del conocimiento del profesor de matemáticas en todos sus dominios, subdominios y categorías y no refiere a ninguna otra ciencia o profesión, entendiendo tal conocimiento profesional como el que el profesor necesita y utiliza (SCHOENFELD, 2010). Por otro lado, se enraíza en la propia matemática, dejando fuera aspectos pedagógicos y psicológicos generales, e incluye las creencias y el dominio afectivo (aunque no son objeto de este trabajo).

Puesto que es un modelo de conocimiento, se consideran aquellos que sustentan las acciones; por ejemplo, se incluye el conocimiento de los procedimientos de resta por compensación 
o descomposición teniendo en cuenta no las instrucciones del algoritmo de restar en sí, sino el cómo se hace, el cuándo puede hacerse, el porqué puede hacerse y las características del resultado obtenido (Figura 1).

Figura 1 - Modelo de Conocimiento Especializado del Profesor de Matemáticas, Mathematics Teachers' Specialized Knowledge, MTSK.

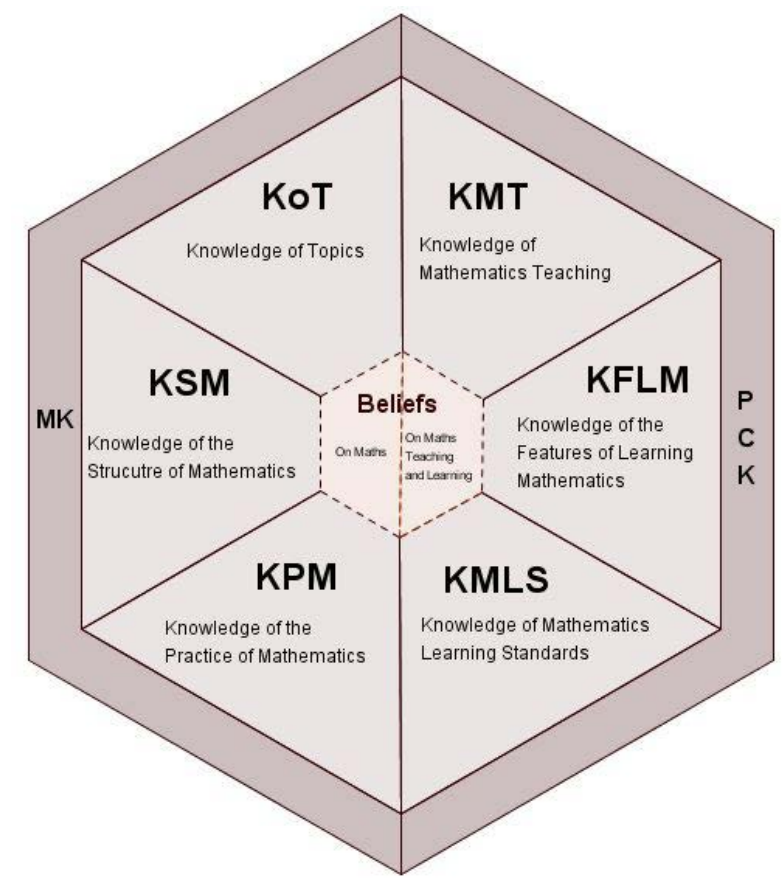

Fuente: Elaboración propia.

Conocimiento Matemático (Mathematical Knowledge, MK):

Integra los conocimientos de la propia disciplina matemática que se enseña, en nuestro caso, la operación resta en los números naturales, y considera tres subdominios: el conocimiento (profundo) del contenido matemático en sí, el conocimiento de las relaciones interconceptuales entre estos conocimientos y el de cómo se produce y procede en matemáticas.

El Conocimiento de los Temas Matemáticos (Knowledge of Topics, KoT ) considera el conocimiento de los contenidos, procesos y procedimientos matemáticos en sí mismos, que se enfoca desde lo que Ma (1999) concibe como el conocimiento profundo de la matemática fundamental. No solo debe conocer los contenidos, procesos y procedimientos escolares, y no solo como disciplina matemática, sino también como elementos interrelacionados, comprendiendo sus propiedades y los procesos que los justifican, sus posibles representaciones, los procedimientos (desde el cómo se hace hasta el cuándo puede hacerse pasando por el porqué), y las diferentes situaciones en las que se presentan. El modelo considera, dentro de este subdominio, las conexiones intraconceptuales, es decir, aquellas relaciones entre conceptos o procesos de un mismo tema.

El Conocimiento de la Estructura Matemática (Knowledge of the Structure of Mathematics, KSM) contempla las conexiones interconceptuales, referidas al conocimiento del profesor sobre las relaciones entre distintos contenidos, ya sean del propio curso, anteriores o posteriores, teniendo en cuenta la temporalidad y dicha interconceptualidad.

El Conocimiento de la Práctica Matemática (Knowledge of the Practice of Mathematics, KPM) se concibe como el conocimiento puesto en juego cuando se realiza una práctica matemática como la resolución de problemas, la definición o demostración de un proceso, el establecimiento de un axioma, el uso correcto del lenguaje y los símbolos, el conocimiento de las condiciones necesarias y suficientes para realizar afirmaciones válidas, entre otras.

Conocimiento Didáctico del Contenido (PCK)

Caracteriza el conocimiento propio de la enseñanza y el aprendizaje del contenido matemático, y tiene importancia desde tres puntos de vista: desde el contenido a enseñar, desde el contenido a aprender y desde la visión general de los estándares del aprendizaje. 
El Conocimiento de las Características del Aprendizaje de las Matemáticas (Knowledge of Features of Learning Mathematics, KFLM) incluye el conocimiento sobre teorías de aprendizaje, sobre las fortalezas y dificultades asociadas al aprendizaje de contenidos concretos, sobre las formas de interacción de los estudiantes con estos, y sobre los intereses y expectativas de los estudiantes con respecto a las matemáticas.

El Conocimiento de la Enseñanza de las Matemáticas (Knowledge of Mathematics Teaching, $K M T$ ) considera el conocimiento de teorías de enseñanza asociadas a un contenido matemático, de los recursos materiales o virtuales para su enseñanza y de las estrategias, técnicas, tareas y ejemplos útiles para ello.

El Conocimiento de los Estándares de Aprendizaje de las Matemáticas (Knowledge of Mathematics Learning Standards, KMLS) considera el conocimiento tanto del currículo oficial vigente en cada país en cada momento, como de estándares definidos por grupos de investigación o asociaciones profesionales de enseñanza y aprendizaje de las matemáticas.

\section{UNA PROPUESTA DE ELEMENTOS DE CONOCIMIENTO ESPECIALIZADO PARA ENSEÑAR LA RESTA EN EDUCACIÓN INFANTIL}

En este apartado presentamos una propuesta de elementos de conocimiento especializado que consideramos que nuestros estudiantes para maestro de Educación Infantil deben movilizar y aprender en relación con la resta en su formación inicial. Circunscribimos el trabajo de la resta al conjunto de los números naturales, tanto en su dimensión conceptual como procedimental. Sin pretensión de ser exhaustivos, hemos seleccionado aquellos aspectos que consideramos que poseen un mayor peso para la comprensión de la resta con vistas a su enseñanza, considerando la totalidad de subdominios según el modelo MTSK.

Para presentar el retrato cohesionado de los conocimientos relacionados con la resta, hemos utilizado la noción de "package of kowledge" de Ma (1999) y lo presentamos en la Figura 2. Esta figura supone responder a la pregunta de qué significa conocer la resta para su enseñanza como profesor de Educación Infantil tanto desde el MK como del PCK. Se trata de una extensión y profundización de la propuesta que presentamos en Ribeiro, Muñoz-Catalán y Liñán (2015) centrada exclusivamente en elementos del MK.

En el centro de la figura hemos partido de la resta como operación matemática en Educación Infantil, la cual debe enmarcarse en la promoción del sentido numérico (e.g., CASTRO; RODRIGUES, 2008), por lo que consideramos que es un contenido que impregna a todo el conocimiento del profesor. Los elementos de conocimiento especializado de los distintos subdominios están incluidos en los rectángulos. Los conocimientos mutuamente relacionados se conectan mediante flechas bidireccionales y aquellas flechas destacadas en negrita señalan la conexión con el centro de la representación. Los números que codifican cada flecha nos servirán para referirnos a las distintas conexiones entre elementos de conocimiento especializado en el desarrollo de nuestra discusión posterior.

Las operaciones con números, tanto para modelar situaciones de la vida real, como para resolver problemas, constituyen una de las grandes ideas que articula el contenido matemático de Educación Infantil (CLEMENTS, 2004). Desde esta perspectiva, la noción de operación, tradicionalmente ligada a las cuatro operaciones clásicas (suma, resta, multiplicación y división) se amplía a otras, que se convierten en el foco de atención en esta etapa educativa y que permiten construir la base adecuada para los aprendizajes futuros en relación con ellas. Así, en sintonía con Clements (2004), las operaciones que están en la base conceptual de la resta se encuentran (KoT) (1): contar, comparar, agrupar y valor de posición, componer/descomponer, repartir equitativamente y añadir/quitar. Contar, como se observa en la Figura 2, juega un papel muy importante en el conocimiento del profesor sobre la resta.

Conocer la resta para su enseñanza significa considerarla como una operación matemática ligada a las demás operaciones básicas, tanto desde una perspectiva conceptual como procedimental (KoT) (2). La complementariedad de la suma y la resta es lo que determina que no se hable de problemas de sumar y restar, sino de problemas aritméticos escolares de estructura aditiva (Carpenter, Fennema, Franke, Levi y Empson, 1999). La resta también está tanto en la base conceptual de la división cuotitiva, o de medida entendida como una sustracción reiterada, como en el sustrato algorítmico de la división y está presente en este procedimiento cuando se valora si 
una determinada cifra del cociente es apropiada, comparando el resultado de multiplicar esta por el divisor con la cantidad del dividendo entre que va a ser dividida. Finalmente, también está presente en la construcción de algunas de las tablas de multiplicar, como la del 9, sobre la base de la del 10 $(9 \times 3=10 \times 3-9)$ y en el procedimiento de la comprobación de la suma (cuando la incógnita está en un sumando) y de la resta (cuando la incógnita está en el sustraendo o en el resultado).

Figura 2 - Elementos de conocimiento especializado del profesor de Educación Infantil en relación con la resta

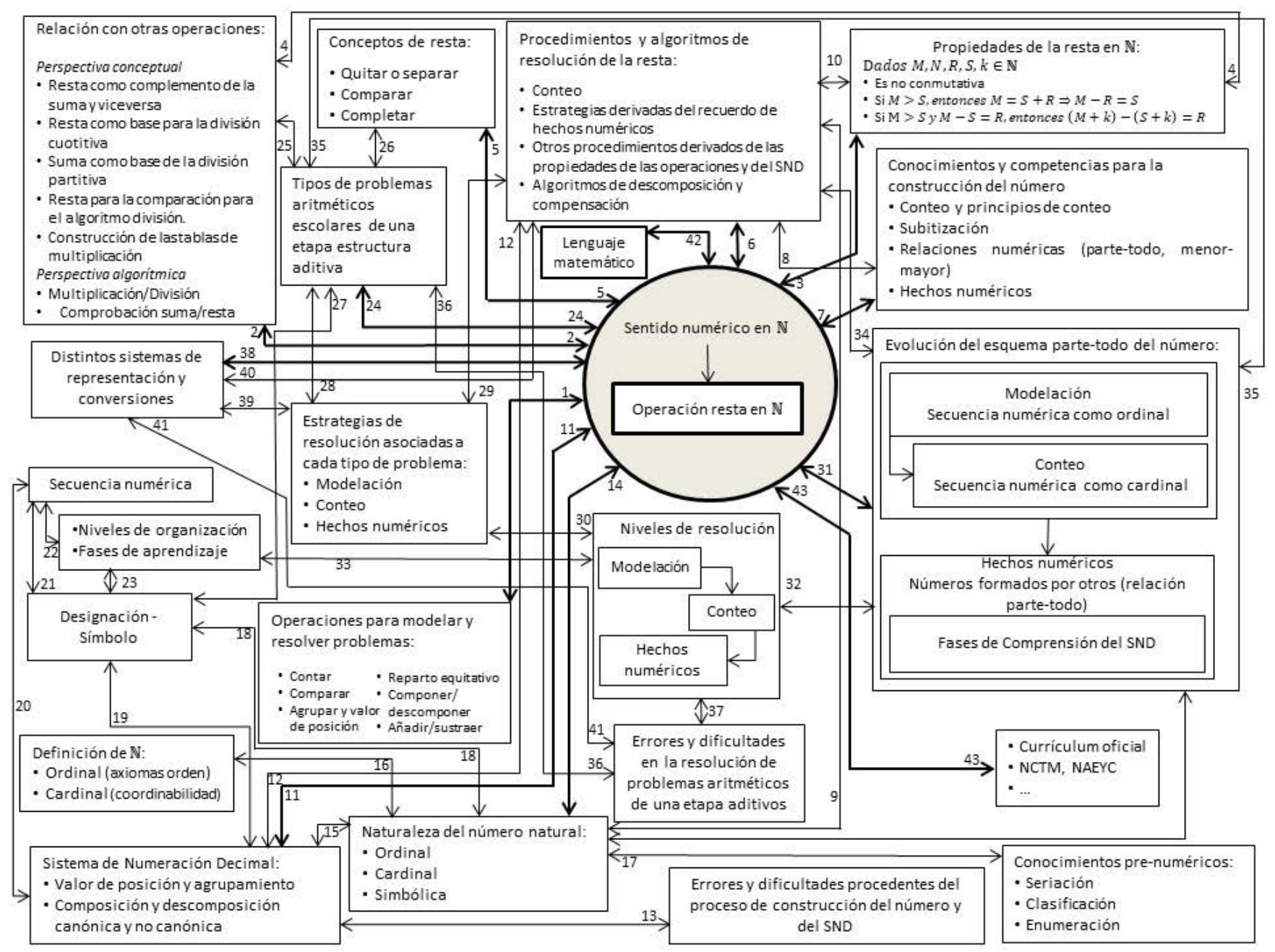

Fuente: Elaboración propia.

Conocer la resta para su enseñanza significa considerarla como una operación matemática ligada a las demás operaciones básicas, tanto desde una perspectiva conceptual como procedimental (KoT) (2). La complementariedad de la suma y la resta es lo que determina que no se hable de problemas de sumar y restar, sino de problemas aritméticos escolares de estructura aditiva (CARPENTER et al., 1999). La resta también está tanto en la base conceptual de la división cuotitiva, o de medida entendida como una sustracción reiterada, como en el sustrato algorítmico de la división y está presente en este procedimiento cuando se valora si una determinada cifra del cociente es apropiada, comparando el resultado de multiplicar esta por el divisor con la cantidad del dividendo entre que va a ser dividida. Finalmente, también está presente en la construcción de algunas de las tablas de multiplicar, como la del 9 , sobre la base de la del $10(9 \times 3=10 \times 3-9)$ y en el procedimiento de la comprobación de la suma (cuando la incógnita está en un sumando) y de la resta (cuando la incógnita está en el sustraendo o en el resultado).

Supone también conocer el conjunto de propiedades que la sustentan (KoT) (3), cómo estas fundamentan la relación con otras operaciones (KSM) (4), así como los distintos significados asociados a la sustracción (retirar, completar y comparar) (KoT) (5). Consideramos que, aunque no conozcan de manera formal las propiedades de esta operación en otros conjuntos de números, como los enteros y los reales, se debe prestar especial atención a cuáles de ellas se verán modificadas. Así, la resta en el conjunto de los naturales no es una operación interna (el resultado de restar dos 
números naturales no siempre es otro número natural), mientras que sí lo es en el conjunto de los números enteros. Es importante esta advertencia porque cuando se extiende el trabajo de la resta a los números enteros es posible efectuar restas en las que el minuendo es menor que el sustraendo , lo que nos induce a la reflexión sobre la importancia que tiene el conocer cómo se debe definir en matemáticas, en este caso, la necesidad de mencionar el conjunto en el que se define la operación (KPM), lo que haría que la definición de la resta en los números naturales sea válida tanto en esta etapa como en las posteriores, cuando ya se tiene en cuenta otros conjuntos de números.

Desde una perspectiva procesual, comprender la resta para su enseñanza implica conocer la diversidad de procedimientos con que se pueden emplear para hallar su resultado (KoT), cada uno de los cuales es esperable en momentos concretos del desarrollo escolar (KMLS) (6). Inicialmente, los procedimientos empleados en esta etapa se basan en determinadas competencias y conocimientos numéricos implicados en el desarrollo de la comprensión del número $(\mathrm{KoT})(7,8)$, como son: el conteo y sus principios (GELMAN; GALLISTEL, 1978; SARAMA; CLEMENTS, 2008), la subitización (CLEMENTS; SARAMA, 2014), relaciones numéricas (parte/todo, menor/mayor) (JUNG, 2011) y hechos numéricos que ponen de relieve el esquema parte/todo del número (RESNICK, 1983). A medida que se va profundizando y ampliando el repertorio de relaciones numéricas, de propiedades de las operaciones y del sistema de numeración decimal, los procedimientos de resolución de la resta se van complejizando, se van volviendo más sofisticados y formales.

Entre estos procedimientos cabe destacar los algoritmos de la resta, como los de descomposición (o "pedir prestado") y el de compensación ("me llevo una"), más extendidos en la cultura española, pero también en otras alrededor del mundo, como puede ser el contexto Brasileño. Por tanto, el conocimiento de la resta y de los fundamentos de estos procedimientos, incluidos los algoritmos, requiere conocer tres elementos con los que están íntimamente relacionados (KSM): el número natural y su triple naturaleza (KoT) $(9,10)$, las propiedades de la resta (KoT) $(10)$ y las propiedades del Sistema de Numeración Decimal (SND) (KoT) (11, 12) (AHARONI, 2008).

Desde la perspectiva de la complejización de la resta como operación, los procedimientos formales o algoritmos requieren necesariamente del conocimiento de nuestro SND (KoT (12). Las reglas para la representación de cantidades que definen nuestro SND repercuten necesariamente en el algoritmo de la resta empleado. En el caso de nuestro SND, los dos principios básicos que lo definen son: el valor de posición (las cifras de un número adquieren su valor en función de la posición; así, la primera cifra de la derecha son las unidades, le siguen las decenas, y así sucesivamente) y el agrupamiento en base 10 (10 unidades de un orden equivalen a 1 unidad de orden superior). La comprensión de ambos principios se requiere para la correcta aplicación de los dos algoritmos y están en la base de las dificultades de los alumnos e incluso de futuros profesores (KFLM) (13) (e.g., MARTINS; RIBEIRO, 2013). Además, el algoritmo de compensación también se apoya en la propiedad compensativa de la resta según la cual la suma de una misma cantidad al minuendo y al sustraendo no altera el resultado (10).

Conocer el SND como conocimiento necesario para resolver la resta en Educación Infantil supone identificar que se trata de un sistema de representación de la noción de número, el cual comienza a desarrollarse en esta etapa. El maestro ha de conocer la triple naturaleza del número (KoT) $(14,15)$ ordinal, cardinal y simbólica, que fundamenta los distintos usos fenomenológicos del número (KoT). También ha de conocer que la dimensión ordinal sustenta la definición del número natural desde la axiomática de Peano, y que la dimensión cardinal es la perspectiva desde la que se define el número al considerar la relación de equivalencia entre conjuntos coordinables (KoT) (16). La base de la construcción del número se sustenta sobre tres conocimientos de naturaleza prenumérica con los que está íntimamente relacionados (17): la seriación, la clasificación y la enumeración. Ha de conocer las características de cada uno de ellos y su relación con el número en sus tres facetas.

EI SND, en cuanto sistema de representación y debido a la naturaleza simbólica del número $(18,19)$, se utiliza para designar cantidades, lo que supone conocer nuestros signos numéricos, su significado (KoT), así como la existencia de un proceso de desarrollo de la función simbólica en los niños al que hay que apoyar y promover (KFLM, KMT), que le lleve a asumir el significado de los signos numéricos (no es fácil asumir, por ejemplo, que cinco caramelos se puede representar con el signo 5, prescindiendo de sus características físicas) y a usarlos siguiendo una gramática matemática. 
Los futuros maestros han de identificar el recitado de las primeras palabras-número (secuencia numérica) como el primer acercamiento al número y al SND (KoT) (20), realizado desde la dimensión ordinal (CLEMENTS, 2004) pues lo que inicialmente perciben los niños es la estabilidad en el recitado de dichas palabras. Como consecuencia de la implicación en actividades de conteo cada vez más complejas, el repertorio de palabras número se va ampliando, se va desarrollando el significado del cardinal del número (FUSON, 1988) y se va identificando el esquema de generación de la lista de palabras número (21). Sin el conocimiento de la construcción de la lista de las palabras-número, no se desarrolla correctamente el pensamiento numérico, pues el conocimiento y comprensión de las reglas de generación de la secuencia derivan en la comprensión de las características del sistema de numeración en el que se incluyen (SND) y viceversa. Por tanto, dos elementos de conocimiento especializado relevante del profesor de Infantil deben ser las fases de aprendizaje de la secuencia numérica (FUSON, 1992) y los niveles de elaboración de la secuencia numérica de palabras número (FUSON; RICHARDS; BRIARS, 1982) (KFLM) (22) y su papel en el proceso de la designación simbólica de cantidades (23).

Otro elemento de este conocimiento especializado sobre la resta son los tipos de problemas aritméticos escolares de una etapa (KoT) (24). Desde la investigación, distintas asociaciones de profesores (NAEYC; NCTM, 2002) señalan la importancia de articular la enseñanza y aprendizaje de la aritmética en los primeros niveles relacionándola con la resolución de problemas aritméticos. Los tipos de problemas atendiendo a un criterio semántico (CARPENTER et al., 1999) permiten construir el significado de la resta en la relación con otras operaciones (KSM) (25) como la suma, dado que ambas operaciones están relacionadas con los problemas de estructura aditiva, y la división, especialmente con los problemas de división cuotitiva, o de medida resolubles mediante restas sucesivas. El problema deberá ser concebido como el medio que permite desarrollar una comprensión significativa del concepto de la operación resta (26) y del significado de los símbolos (27) (KoT, KMT). Así, por ejemplo, los problemas de tipo Cambio contribuyen a construir el significado de la resta ligado a "quitar o separar", los de combinación con incógnita en una parte ayudan al concepto de resta como "completar" y los problemas de comparación potencian el significado de resta como "comparar".

Para comprender cómo piensan los niños sobre la resta, es preciso identificar la relación bidireccional entre los tipos de problemas y las estrategias de resolución que los alumnos utilizan (CARPENTER et al., 1999). No nos referimos a las estrategias que el profesor proporciona al alumno y éste elige a conveniencia, sino de procedimientos naturales que los alumnos manifiestan en su empeño por comprender la situación de cada tipo de problema y tratan de resolverlo (KoT) (28, 29). Así, por ejemplo, la estrategia "contar hasta" es específica de los problemas de cambio crecer, en los que la incógnita está en el cambio. Según los trabajos de Carpenter y sus colaboradores (BEBOUT; CARPENTER, 1989; CARPENTER et al., 1999) estas estrategias se pueden organizar en tres tipos diferentes (KoT): estrategias de modelación, estrategias de conteo y estrategias de hechos numéricos. En las estrategias de modelación los niños necesitan representar tanto las cantidades como la acción, obteniendo el resultado contando uno a uno los objetos resultantes. En las estrategias de conteo se trabaja con una representación mental más que física de los objetos y se obtiene el resultado mediante un doble conteo simultáneo (el propio de la secuencia numérica y el de la cantidad de números recitados) por lo que el uso de los contadores permite llevar la cuenta del número de palabras recitadas en la secuencia de conteo. Las estrategias de hechos numéricos son independientes de la estructura del problema y se resuelven utilizando hechos numéricos y hechos derivados de estos, los cuales se construyen a partir de la comprensión de relaciones numéricas y se sustentan en el sentido numérico. En su proceso de aprendizaje, todos los alumnos van pasando por estos tipos de estrategias, pudiendo establecerse tres niveles de desarrollo, cuyo nombre coincide con el tipo de estrategias empleado: nivel de modelación, nivel de conteo y nivel de hechos numéricos (KFLM) (30). Así, dado el problema: "María tiene 6 caramelos, su madre le da algunas y al final tiene 13", un alumno que esté en el nivel de modelación lo resolverá representando con fichas el número 6, irá añadiendo fichas hasta llegar a 13, aunque ubicándolas en un lugar separado de la cantidad inicial y contará de una en una las fichas añadidas (estrategia: añadir hasta). En niño que esté en el nivel de conteo dirá: " $6 . .7,8,9,10,11,12$ y 13 ", con cada número recitado desde el 7 levanta un dedo, observando al final que ha levantado 7 dedos (estrategia: contar hasta). Un niño 
que esté en el nivel de hechos numéricos podrá decir "7, porque 6 y 6 son 12 y una 7", basándose en su conocimiento del doble del 12.

Un profesor de Educación Infantil ha de saber que lo que condiciona que un niño esté en un nivel u otro es su comprensión del número $(\operatorname{KFLM})(31,32)$. El proceso de comprensión del número puede entenderse como la evolución del esquema parte-todo del número (RESNICK, 1983), que va pasando por distintos hitos. Este desarrollo se inicia con la asimilación de la componente ordinal del número, por lo que la producción de las etiquetas numéricas en orden es clave para iniciar el proceso de conteo (nivel de modelación). El siguiente hito es el desarrollo del significado cardinal de las palabras números, lo que les permite cortar la secuencia numérica y usar los números como contadores (nivel de conteo) (Fuson, 1988). Se comienza a desarrollar a nivel de cadena cortable y numerable de los niveles de elaboración de la secuencia numérica de palabras número (FUSON; RICHARDS; BRIARS, 1982) (KFLM) (33). El inicio del desarrollo del esquema parte-todo como tal se produce cuando observan que los números están formados por otros (e. g. $7=4+3=3+3+1$ ), lo que les permite descomponerlos y tener una mayor flexibilidad para el cálculo, basándose en el conocimiento de hechos y relaciones numéricas. En esta fase, la sucesión de números se asocia a una secuencia lineal infinita (nivel de hechos numéricos). Cuando el dominio de números se va ampliando, se comienza comprender el número como elemento articulador del Sistema de Numeración Decimal y, a nivel conceptual, la sucesión de números se articula en forma de filas y columnas (lo que permite sumar tanto de 1 en 1, como de 10 en 10, 100 en 100, y así sucesivamente). Esta nueva etapa está formada por tres fases (RESNICK, 1983), en la última de las cuales se consigue comprender los fundamentos de los algoritmos de la resta (34). El conocimiento de esta evolución permite al profesor seleccionar y secuenciar los problemas más apropiados (KMT) (35) que va a trabajar en función de sus expectativas de aprendizaje y anticipar posibles dificultades en función de los problemas propuestos (KFLM) (36) y del nivel de comprensión del número que posea el alumno (37).

Un profesor debe conocer también el tipo de sistemas de representación (símbolo escrito, lenguaje hablado, situaciones reales, gráficos y modelos manipulativos) que puede utilizar en el aula de Infantil para promover la comprensión de la resta, así como el potencial que posee el uso variado de ellos y la conversión entre ellos, en sintonía con el Modelo de traslaciones entre múltiples representaciones (LESH; POST; BEHR, 1987) (KMT) (38). En particular, en lo que a modelos manipulativos se refiere, un profesor debe conocer qué recursos se pueden utilizar para que los alumnos resuelvan la resta modelizando, con sus potencialidades y limitaciones (KMT). Deben saber valorar qué sistemas de representación y conversiones entre ellos son más apropiados para cada etapa de comprensión del número (39), para cada tipo de algoritmo (40) y en función de los errores particulares que presenten los alumnos (41).

El profesor de Educación Infantil presenta un reto especial con respecto al lenguaje (42), en una etapa generadora del mismo. El lenguaje es a la vez sistema de representación de conceptos, en el sentido de Lesh, Post y Behr (1987) (KoT) y recurso para la enseñanza (KMT), pero también juega un papel muy importante en relación con los símbolos y el uso del lenguaje matemático formal (KPM). El profesor ha de conocer el lenguaje matemático preciso y apropiado asociado a todos los conceptos y procedimientos relacionados con la resta. Este lenguaje ha de ser siempre correcto, aunque no necesariamente debe contener la amplitud y profundidad del concepto considerado, el cual se irá enriqueciendo y ampliando en cursos posteriores. Ejemplos de lenguaje no completo e incorrecto sería afirmar que el minuendo nunca puede ser menor que el sustraendo, o que al dividir siempre obtengo una cifra menor, resultados solo válidos en el contexto de los números naturales, pero no válidos al ampliar la noción de número y de operación (recordemos que en la definición de operación se debe elegir el conjunto en el que está definida). Otro ejemplo de lenguaje incorrecto aparece cuando se consideran los dígitos del minuendo como amigos que se prestan lo que necesitan en el caso del algoritmo de la descomposición; además de ser matemáticamente falso, puede llevar a interpretar el número como cifras independientes sin relación entre sí (MA, 1999) y podría considerarse un posible obstáculo didáctico.

Finalmente, un profesor debe conocer lo prescrito en los currículos oficiales sobre la resta en cada etapa (no solo en los documentos oficiales de Infantil), pero también lo que procede de investigaciones o de asociaciones profesionales como la National Council of Teachers of Mathematics (NCTM) o la National Association for the Education of Young Children (NAEYC) (NAEYC; NCTM, 2002; NATIONAL COUNCIL OF TEACHERS OF MATHEMATICS, 2000) (KMLS) (43). 


\section{REFLEXIONES SOBRE LA NATURALEZA DEL CONOCIMIENTO ESPECIALIZADO DEL PROFESOR DE INFANTIL Y CONCLUSIONES FINALES}

En el anterior apartado hemos presentado una propuesta de elementos de conocimiento especializado sobre la resta que consideramos que se deben trabajar en la formación inicial (y continua) de los profesores de Educación Infantil, sobre la base de nuestras investigaciones en la etapa y de nuestra experiencia como formadores de estos profesionales. En este apartado mostramos nuestras reflexiones sobre las características de este conocimiento en relación con la resta, pero también de aspectos que comienzan a emerger sobre la naturaleza del conocimiento de este profesional de manera general.

En la Figura 2 se pone de relieve dos aspectos que parecen definir el conocimiento especializado de este profesional en relación con la resta: la densidad y cohesión, tanto por la riqueza de elementos de conocimientos que lo constituye como por las conexiones que se establecen entre ellos. La densidad no solo aparece representada en esta figura por el número de elementos incluidos en los rectángulos, sino también hay conocimiento expresado a través de las flechas que conectan estos elementos de conocimientos. En la propuesta de elementos de conocimiento para la resta que acompaña a la Figura 2, es posible identificar nuevas relaciones, pero hemos seleccionado aquellas que consideramos potentes, articuladoras y definitorias de este conocimiento especializado del profesor de Infantil.

Las conexiones son de tres tipos, cada una de las cuales se corresponde con un subdominio del MTSK (CARRILLO et al., 2013). Hay flechas que se corresponden con conexiones intraconceptuales y que, por tanto, son elementos del Conocimiento de los Temas (Knowledge of Topics, KoT) como la conexión 12, que conecta los tipos de algoritmos y el Sistema de Numeración Decimal, ya que éste y sus propiedades permiten conocer por qué funcionan aquellos. Otro ejemplo lo encontramos en la conexión 26, que relaciona los tipos de problemas de estructura aditiva con los significados de la resta que cada uno potencia. No obstante, la mayor parte de elementos de este subdominio se encuentra representada en los rectángulos.

Situación opuesta encontramos con el subdominio del Conocimiento de la Estructura Matemática (Knowledge of the Structure of Mathematics, KSM) que, debido a su naturaleza relacional, solo se puede representar mediante flechas. Estas flechas expresan relaciones interconceptuales. La figura es muy rica en este tipo de conocimiento y consideramos que puede ser uno de los subdominios que podría definir la naturaleza del conocimiento especializado de esta etapa. Un profesor que sea consciente de las repercusiones que para el conocimiento de la resta posee trabajar en $\mathrm{N}$ en Infantil (3) y cómo algunas de estas características cambian cuando en Primaria trabajen con otros conjuntos de números puede llevarle a no reforzar la idea de que el minuendo ha de ser siempre mayor que el sustraendo e, incluso, a proponer problemas con esta situación con el fin de fomentar el diálogo en la clase y de que busquen distintas soluciones al problema. Un profesor que considere la resta en su relación con la división (2) (conexión de complejización) puede llevarle a tomar la decisión de priorizar el algoritmo de la resta por compensación porque, a pesar de su mayor grado de sofisticación, es más potente que el de descomposición para el algoritmo de la división. El profesor de Educación Infantil debe saber la relación que existe entre la resta en su perspectiva procedimental y la enumeración (17) (conexión de simplificación). Este contenido suele estar ausente en los programas escolares, pero un profesor que es consciente de esta relación propondrá actividades de enumeración desde los 3 años de edad que ayude a los alumnos a construir una sólida base para enfrentarse con éxito a situaciones de conteo. Estas conexiones interconceptuales ponen de relieve la génesis epistemológica en el aprendiz, esto es, el papel generativo que cada contenido juega en la construcción de los demás en situación de aprendizaje y otorga una entidad diferente a la resta como objeto de enseñanza y aprendizaje en esta etapa.

Un tercer tipo de conexiones son las que se establecen entre elementos del Conocimiento Matemático (Mathematical Knowledge, MK) con los del Conocimiento Didáctico del Contenido (Pedagogical Content Knowledge, PCK). Estas son especialmente interesantes en el sentido de que los elementos del PCK hunden sus raíces en elementos del MK (OPPERMANN; ANDERS; HACHFELD, 2016), perdiendo su razón de ser si no están ligados a estos.

Cuando en este trabajo hemos extendido el trabajo que iniciamos en Ribeiro, Muñoz-Catalán y Liñán (2015) para incluir elementos del PCK hemos observado que el peso de los elementos del MK seguía siendo relevante en el conocimiento especializado sobre la resta. Basándonos en nuestra 
experiencia como formadores de maestros de Infantil, esta observación puede ser extensible a cualquier contenido matemático. Este hecho contrasta con la perspectiva, a menudo bastante limitada y estrecha, que se tiene sobre el MK del profesor de Educación Infantil (MEWBORN, 2001), que se corresponde con el hecho de que no se les suele exigir a lo largo de su carrera profesional una formación profunda y específica en matemáticas (OPPERMANN; ANDERS; HACHFELD, 2016). De hecho, en la mayoría de universidades españolas (algo similar se puede decir también de otros contextos, incluyendo Brasil), de los 240 créditos de los que consta el plan de estudio del profesor de Educación Infantil, solo 6 créditos obligatorios se imparten específicamente desde la Educación Matemática. Una propuesta de este tipo pretende contribuir a la necesaria reflexión sobre el conocimiento deseable de este profesional e impulsar, con argumentos sólidos, una formación didáctico-matemática más robusta.

Nuestra propuesta de elementos de conocimiento especializado para la resta en Educación Infantil pone de relieve los tres aspectos siguientes: por un lado, una relación particular entre el contenido que el alumno aprende y el conocimiento especializado del profesor de Infantil, siendo esta, quizás, la etapa educativa en la que esa identificación es menos evidente o aparentemente más distante. Por ejemplo, en el caso de la designación matemática (CHAMORRO, 2005), mientras los alumnos se enfrentarán a actividades donde tengan que designar elementos sin ser conscientes de ello (e.g., procesos, clases, conjuntos, algoritmos), la visión profesional de los profesores debe llevarlos a ver que están sentando las bases para el uso significativo de símbolos, que les va a permitir adoptar los números como representantes de conjuntos de objetos dotados con la cantidad que cada signo indica, con los que a su vez podrán construir la secuencia numérica, para efectuar, a continuación, conteos y poder operar en situación de sustracción. El profesor de Infantil que posee esta visión cohesionada de la resta será capaz de promover un aprendizaje más interconectado e integrado en sus estudiantes, especialmente eficaz para los aprendizajes posteriores (NAEYC; NCTM, 2002) y para facilitar una educación matemática continua (FERNÁNDEZ et al, 2011) entre etapas.

Por otro lado, junto al conocimiento de aspectos matemáticos de la resta en $\mathrm{N}$, la propuesta ubica en un lugar relevante el conocimiento sobre las fases que los niños siguen en su proceso de comprensión del número (31) que pertenece al subdominio de Conocimiento de Características del aprendizaje de las matemáticas (Knowledge of Features of Learning Mathematics, KFLM). Este conocimiento le permite anticipar el tipo de estrategia que va a emplear, el nivel en que se encuentra, qué tipo de problemas puede resolver y cuáles no, en qué conocimientos ha de incidir, lo que le llevará a disponer de argumentos necesarios para crear oportunidades de aprendizaje ajustadas a cada niño. Este elemento, por ejemplo, pone de relieve otra característica que puede ser diferenciadora del conocimiento de este profesional respecto del profesor de etapas posteriores y es el importante papel del conocimiento del alumno de Infantil y de su proceso de aprendizaje. Parece que las características psicológicas y evolutivas de los alumnos de esta etapa tienen repercusiones importantes en la articulación del conocimiento especializado del profesor sobre la resta en esta etapa.

Finalmente, la propuesta presentada muestra un posicionamiento particular sobre el enfoque que la resta ha de adoptar en esta etapa, centrada en su aspecto conceptual, ligada a los contextos que permitan dotarles de significado y a la importancia del dominio del conteo, del principio de cardinalidad, del dominio de hechos numéricos y de la familiarización con relaciones numéricas (CARPENTER et al., 1999; FUSON, 1998; JUNG, 2011; SARAMA; CLEMENTS, 2008) (7). Esta consideración resuelve una carencia que Parks y Wager (2015) identifican en las publicaciones sobre propuestas formativas para estos profesionales en revistas relevantes del área, las cuales se centran exclusivamente en las operaciones desde su enfoque procedimental formal lo que "ha limitado las oportunidades de los estudiantes para maestros para aprender sobre tópicos importantes relacionados con los números para niños de preescolar y jardín de Infancia, como contar y cardinalidad, composición y descomposición de números, y relaciones numéricas" (p. 132).

El modelo MTSK (CARRILLO et al, 2013; CARRILLO et al., 2017; MUÑOZ-CATALÁN et al., 2015) se ha mostrado útil como herramienta analítica para reflexionar sobre el conocimiento especializado deseable del profesor de Infantil en relación con la resta. Percibimos ese potencial, principalmente, en lo referente al MK, al promover la reflexión sobre elementos del KoT, destacar el papel de la cohesión de ese conocimiento a través del KSM y, finalmente, por destacar un 
subdominio que, tradicionalmente, ha pasado desapercibido para este profesional: El Conocimiento de las Prácticas Matemáticas (Knowledge of the Practice of Mathematics, KPM), que en la propuesta que hemos presentado está representado principalmente a través del lenguaje. Al ser la etapa de Educación Infantil generadora de lenguaje, los profesores han de ser especialmente cuidadosos con la precisión de los términos utilizados si son conscientes del papel que los símbolos y este lenguaje juega en la creación y validación del conocimiento matemático. En la propuesta de conocimiento especializado sobre la resta que presentamos en Muñoz-Catalán, Gavilán-Izquierdo y Liñán (en presa) para el profesor de Educación Primaria emergió otro elemento relacionado con la práctica matemática: la algoritmización en su dimensión horizontal y vertical. Este subdominio abre una vía de indagación para identificar cuáles son las prácticas matemáticas características de esta etapa y, por tanto, qué conocimiento debe tener el profesor de Educación Infantil respecto de ellas.

La propuesta de elementos de conocimiento especializado sobre la resta del profesor de Educación Infantil que hemos presentado supone una ampliación de la noción de "package of knowledge" utilizada por Ma (1999), en la que integramos aspectos del PCK e incluye tanto aspectos propuestos por los profesores de su estudio, como aspectos que incorporamos a la luz de nuestra experiencia docente e investigadora. En sintonía con ella, consideramos que puede considerarse como una estrategia potente de reflexión en la formación inicial y continua porque permite organizar el conocimiento de la formación de una manera cohesionada y sus elementos son inspiradores de nuevos elementos del conocimiento. Desde esta perspectiva, por ejemplo, asumir que existen una serie de conocimientos y competencias básicas para la construcción del número (7) supone un incentivo para que los profesores reflexionen sobre estrategias, tareas, recursos, ejemplos que promuevan cada uno de ellos (Knowledge of Mathematics Teaching, KMT)

Nuestro reto investigador ahora es utilizar esta herramienta como base para una propuesta de formación de profesores, que nos permita contrastarla y enriquecerla, a la misma vez que promovemos el desarrollo de este tipo de conocimiento. En esta línea, nos interesa indagar sobre cómo se aprenden y enseñan en la formación de maestros y cómo se traslada ese conocimiento al aula de Educación Infantil. Sería interesante proponer experiencias muy ligadas a la propia práctica (considerada de forma amplia), es decir, situaciones con las cuales los profesores se pudieran identificar y les permitieran explorar y desarrollar su conocimiento especializado. También habría que considerar experiencias que les permitieran enfrentarse a los mismos tipos de dificultades que sus alumnos pueden presentar (e.g., JAKOBSEN; RIBEIRO; MELLONE, 2014; PINTO; RIBEIRO, 2013; TIROSH et al. 2011) -por supuesto, a niveles distintos-, de modo que consigan, posteriormente, entender mejor las raíces de las mismas y sean capaces de gestionarlas eficazmente en situaciones de contingencia (ROWLAND; HUCKSTEP; THWAITES, 2005). Pero, para tal fin, deberíamos intentar hacer partícipes a los profesores en el diseño de los programas de formación, principalmente a los profesores en activo. Consideramos que es importante potenciar encuentros de reflexión y concienciación de los propios formadores de profesores relativa a la responsabilidad de cada uno de nosotros. Esa reflexión se deberá basar, entre otros, en el hecho de que el mismo tipo de lógica que se discute respecto a la importancia del conocimiento especializado del profesor como un factor esencial para los aprendizajes de los alumnos (e.g., NYE; KONSTANTOPOULOS; HEDGES, 2004), se aplique a cada uno de nosotros, como formadores de profesores, siendo nuestros alumnos los propios profesores o estudiantes para profesores (JAWORSKI, 2008). En ese sentido, el conocimiento del profesor sobre la resta ha resultado un desencadenante de reflexión sobre nuestra propia práctica, siendo esta una línea de trabajo complementaria a seguir para potenciar las relaciones efectivas entre teoría, práctica y formación.

\section{Agradecimientos}

Financiado por el proyecto «Caracterización del conocimiento especializado del profesorado de Matemáticas» (EDU2013-44047-P, Ministerio de Economía y Competitividad, España), y por el Centro de Estudios Universitarios Cardenal Spínola CEU de Sevilla (España). 


\section{REFERENCIAS}

AHARONI, R. Aritmética para Pais. Lisboa: Gradiva. 2008.

BEBOUT, H. C.; CARPENTER, T. P. Assessing and building thinking strategies: Necessary bases for instruction. En: TRAFTON, P. R.; SHULTE, A. P. (Eds.), New directions in elementary school mathematics (yearbook of the National Council of Teachers of Mathematics), p. 59-69. Reston, VA: National Council of Teachers of Mathematics. 1989.

CARPENTER, T. P. et al. Children's Mathematics. Cognitively Guided Instruction. Portsmouth, $\mathrm{NH}$ : Heinemann. 1999.

CARRILLO, J. et al. Defining specialized knowledge for mathematics teaching. In B. Ubuz, Ç. Haser y M. A. Mariotti (Eds.), Proceeding of CERME8, p. 29852994. Ankara, Turkey: Middle East Technical University. 2013.

CARRILLO, J. et al. Les connaissances duprofesseur dans une perspective basée sur leur spécialisation: MTSK. Annales de didactique et de sciences cognitives, v. 22, 185 - 205. 2017.

CASTRO, J. P. ; RODRIGUES, M. Sentido de número e organização de dados, Textos de Apoio para Educadores de Infância. Lisboa: ME-DGIDC. 2008.

CHAMORRO, M. C. (Coord). Didáctica de las matemáticas. Educación Infantil. Madrid: Pearson. 2005.

CLEMENTS, D. H. Major Themes and recommendations. In: CLEMENTS, D.H., SARAMA, J.; DIBIASE, A.-M. (Eds.), Engaging Young Children in Mathematics. Standards for Early Childhood Mathematics Education, p. 7-72. Mahwah, NJ: Lawrence Erlbaum Associates. 2004.

CLEMENTS, D. H.; SARAMA, J. Learning and teaching early math: The learning trajectories approach. New York: Routledge. 2014.

CONTRERAS, L.C. et al. Un Estudio Exploratorio sobre las Competencias Numéricas de los Estudiantes para Maestro. Boletim de Educaçao Matematica, 26 (42b), 433-458. 2012.

FERNANDEZ, S.; FIGUEIRAS, L. El conocimiento del profesorado necesario para una educación matemática continua. En: MORENO, M.M. et al. (Eds.), Investigación en Educación Matemática XIV (pp. 291-301). Lleida: SEIEM. 2010.

FERNÁNDEZ, S. et al. Re-defining HCK to approach transition. In: PYTLAK M.; ROWLAND, T.; SWOBODA, E. (Eds.), Proceedings of CERME 7, p. 2640-2649. Rzeszów, Poland University of Rzeszów. 2011.

FUSON, K. C. Children's counting and concepts of numbers. New York: Springer-Verlag. 1988.

Research on whole number addition and subtraction. En: GROUWS, D. (Ed.), Handbook of Research on Mathematics Teaching and Learning, p. 243-275. New York: Macmillan \& Co. 1992.

FUSON, K.C.; RICHARDS, J.; BRIARS, D.J. The acquisition and elaboration of the number word sequence. En: BRAINERD, C. (Ed.), Progress in cognitive development research: Vol 1. Chilcren's logical and mathematical cognition, p.33-92. New York: Springer-Verlag. 1982.

GELMAN, R.; GALLISTEL, C.R. The child's understanding of number. Cambridge: Harvard University Press. 1978.

HIEBERT, J.; GROUWS, D. The effects of classroom mathematics teaching on students' learning. In F. Lester (Ed.), Handbook of Research on Mathematics Teaching and Learning, p. 371-404. NCTM: Information Age Publishing. 2007.

HILL, H. C.; ROWAN, B.; BALL, D. L. Effects of teachers' mathematics knowledge for teaching on student achievement. American Education Research Journal, v. 42, n. 2, p. 371-406. 2005.

JAKOBSEN, A.; RIBEIRO, M.; MELLONE, M. Norwegian prospective teachers' MKT when interpreting pupils' productions on a fraction task. Nordic Studies in Mathematics Education, v. 19, n 3-4, p. 135-150. 2014.

JAKOBSEN, A.; THAMES, M. H.; RIBEIRO, C. M. Delineating issues related to Horizon Content Knowledge for mathematics teaching. In: UBUZ, B. HASER, Ç. ; MARIOTTI, M. A. (Eds.), Proceedings of CERME 8, p. 3125-3134. Antalia, Turkie: ERME. 2013.

JAWORSKI, B. Mathematics teacher educator learning and development. In: JAWORSKI, B.; WOOD, T. (Eds.), International handbook of mathematics teacher education, v. 4, p. 1-13. Rotterdam: Sense. 2008.

JUNG, M. Number Relationships. A Preschool Classroom. Teaching Children Mathematics, v. 17, n. 9, 550557. 2011.

KAMII, C.; LEWIS, B.; KIRKLAND, L. Fluency in subtraction compared with addition. Journal of Mathematical Behaviour, v. 20, p. 33-42. 2001.

LEE, J. Exploring kindergarten teachers' pedagogical content knowledge of mathematics. International Journal of Early Childhood, v. 42, p. 27-41. 2010. 
LESH, R.; POST, T.; BEHR, M. Representations and translations among representations in mathematics learning and problem solving. En: JANVIER, C. (Ed.), Problems of representation in the teaching and learning of mathematics, p. 33-40. Hillsdale, NJ: Lawrence Erlbaum. 1987.

MA, L. Knowing and teaching elementary mathematics: Teachers' understanding of fundamental mathematics in China and the US. Mahwash, NJ: Lawrence Erlbaum Associates, Publishers. 1999.

MARTINS, F.; RIBEIRO, C. M. Atribuir sentido aos raciocínios associados às resoluções de alunos no caso da subtração: discutindo o conhecimento de futuros professores. In: CADIMA et al. (Eds.), Atas da Conferência Internacional de Investigação, Práticas e Contextos em Educação, p. 192-200. Leiria: ESECS. 2013.

MCCRAY, J.; CHEN, J.Q. Pedagogical content knowledge for preschool mathematics: Construct validity of a new teacher interview. Journal of Research in Childhood Education, v. 26, p. 291-307. 2012.

Mewborn, D. Teachers content knowledge, teacher education, and their effects on the Preparation of elementary teachers in the United States. Mathematics Education Research Journal, 3, 28-36. 2001.

MUÑOZ-CATALÁN, M.C. et al. Conocimiento especializado del profesor de mate-máticas (MTSK): un modelo analítico para el estudio del conocimiento del profesor de matemáticas. La Gaceta de la RSME, v. 18, n. 3, 589-605. 2015.

MUÑOZ-CATALÁN, M.C.; GAVILÁN, J.M.; LIÑÁN, M. M. (en prensa). La escritura como recurso para promover el conocimiento especializado del estudiante para maestro en matemáticas. En E. Gallardo y F. Núñez (Coords.), Escribir en las disciplinas: intervenciones para desarrollar los géneros académicos y profesionales en la Educación Superior. Editorial Síntesis.

NAEYC; NCTM. National Association for the Education of Young Children and National Council for Teachers of Mathematics. Early childhood mathematics: Promoting good beginnings. A joint position statement. 2002. Disponible en: <http://www.naeyc. org/files/naeyc/file/positions/psmath.pdf>.

NATIONAL COUNCIL OF TEACHERS OF MATHEMATICS. Principles and standards for school mathematics. Reston, VA: The Council. 2000.

NYE, B.; KONSTANTOPOULOS, S.; HEDGES, L. V. How large are teacher effects? Educational Evaluation and Policy Analysis, v. 26, n. 3, p. 237-257. 2004.

OPPERMANN, E.; ANDERS, Y.; HACHFELD, A. The influence of preschool teachers' content knowl- edge and mathematical ability beliefs on their sensitivity to mathematics in children's play. Teaching and Teacher Education, v. 58, p. 174-184. 2016.

PARKS, A.N.; WAGER, A. A. What Knowledge is Shaping Teacher Preparation in Early Childhood Mathematics? Journal of Early Childhood Teacher Education, v. 36, n. 2, p. 124-141. 2015.

PINTO, H.; RIBEIRO, C. M. Conhecimento e formação de futuros professores dos primeiros anos - o sentido de número racional. Da Investigação às Práticas, v. 3, n. 1, 85-105. 2013.

RESNICK, L. A developmental theory of number understanding. En: GINSBURG, H. (Ed.), The development of mathematical thinking, p. 109-151. New York: Academic Press. 1983.

RIBEIRO, C.M.; MUÑOZ-CATALÁN, M.C.; LIÑÁN, M. M. Discutiendo el conocimiento matemático especializado del profesor de Infantil como génesis de aprendizajes futuros. En: GÓMEZ-CHACÓN, I. et al. (Eds.). MWS, Proceedings Fourth ETM Symposium. p. 575-589. Madrid: Universidad Complutense de Madrid. 2015.

ROWLAND, T.; HUCKSTEP, P.; THWAITES, A. Elementary teachers' mathematics subject knowledge: the knowledge quartet and the case of Naomi. Journal of Mathematics Teacher Education, v. 8, p. 255281. 2005.

SARAMA, J.; CLEMENTS, D. H. Mathematics in early childhood. En: SARACHO, O.; SPODEK, B. (Eds.), Contemporary Perspectives on Mathematics in Early Childhood Education, p. 67-94. Charlotte, NC: Information Age Publishing. 2008.

SCHOENFELD, A.H.; KILPATRICK, K. Toward a theory of proficiency in teaching mathematics. In: TIROSH, D.; WOOD, T. (Eds.), The international handbook of mathematics teacher education: Tools and processes in mathematics teacher education, $\mathrm{v}$. 2, p. 321-354. Rotterdam: Sense Publishers. 2008.

SCHOENFELD, A. H. How we think. Nueva York: Routledge. 2010.

SHULMAN, L.S. Those who understand: Knowledge growth in teaching. Educational Researcher, v. 15, n. 2, 4-14. 1986.

Knowledge and teaching: Foundations of a new reform. Harvard Educational Review, v. 57, n. 1, 1-21. 1987.

THAMES, M.; BALL, D. What mathematical knowledge does teaching require? Teaching Children Mathematics, v. 14, n. 4, p. 220-229. 2010. 
TIROSH, D. et al. From preschool teachers' professional development to children's knowledge: comparing sets. Journal of Mathematics Teacher Education, v. 14, p. 113-131. 2011.

PARKS, A.N.; WAGER, A.A. What Knowledge is Shaping Teacher Preparation in Early Childhood Mathematics? Journal of Early Childhood Teacher Education, v. 36, n. 2, p. 124-141. 2015. 